\title{
Medium adsorbance fraction of reticulocyte and myeloperoxidase index may individuate a patient subset with a low risk of chemotherapy-related neutropenia
}

\author{
GIULIO GIORDANO ${ }^{1}$, PIER FRANCESCO FERRUCCI ${ }^{4}, \mathrm{CHIARA} \mathrm{NICCI}^{1}$, \\ TIZIANA GRAFONE ${ }^{1}$, ROSA TAMBARO ${ }^{2}$, STEFANO PAPINI $^{3}$, GIULIANA FARINA ${ }^{1}$, \\ SILVIA PIANO $^{1}$, BRUNO ZAPPACOSTA $^{3}$ and SERGIO STORTI ${ }^{1}$ \\ ${ }^{1}$ Onco-Haematology Division, ${ }^{2}$ Oncology Division, ${ }^{3}$ Laboratory Medicine Division, Centre for High \\ Technology Research and Education in Biomedical Sciences 'John Paul II', Catholic University, \\ Campobasso; ${ }^{4}$ Melanoma and Sarcoma Division, Istituto Europeo di Oncologia, Milan, Italy
}

Received February 1, 2008; Accepted May 30, 2008

DOI: 10.3892/or_00000207

\begin{abstract}
In neoplastic patients chemotherapy frequently involves severe myeloid suppression. Sometimes myeloid suppression is the main cause of therapy recycling delay with severe and prolonged neutropenia, anaemia and thrombocytopenia. Our study aimed to verify whether there is a correlation between reticulocyte fractions, reticulocyte indices, myeloperoxidase index (MPXI) and post-chemotherapy myelopoietic function and severe post-chemotherapy neutropenia. A cohort of 112 patients was identified, 30 with lymphoma or myeloma and 82 with solid neoplasms with bone marrow micrometastases. The patients were treated with chemotherapy (CT). After CT, 60 patients had neutropenia (ANC $<500 / \mathrm{mcl}$ ) for a median of 7 days (range 3-21). Before CT, myelopoietic function was assessed by the abovementioned parameters using a hematologic automated analyzer. We assigned patients with an MPXI-positive value and medium adsorbance fraction of reticulocyte (MFR) $>10.7 \%$ a score of 1 , and a score of 0 was assigned to the remaining patients. Patients with a score of 1 showed a lower number of neutropenic events (only 9 out of 36 patients) than those with a score of 0 (51 out of 76 patients), $\mathrm{p}<0.0001$. MPXI and MFR may be used in the assessment of myelopoiesis before CT administration, independently of the type of tumor, CT regimen and number of CT cycle, with the aim of identifying a patient subset with a lower risk of developing neutropenia post-CT.
\end{abstract}

Correspondence to: Dr S. Storti or Dr G. Giordano, OncoHaematology Division, Centre for High Technology Research and Education in Biomedical Sciences 'John Paul II', Catholic University, 86100 Campobasso, Italy

E-mail: sstorti@rm.unicatt.it

E-mail: giulio.giordano@rm.unicatt.it

Key words: chemotherapy, neutropenia, myeloperoxidase index, medium absorbance fraction of reticulocyte

\section{Introduction}

The aging of populations makes neoplasia one of the main causes of morbidity and mortality in Western countries. Neoplasias often require, mainly in onco-hematology and in general oncology, aggressive treatments, including chemotherapy.

Chemotherapy, in neoplastic patients, frequently involves severe myelosuppression (1). After high-dose chemotherapy, $30-50 \%$ of patients will not experience febrile neutropenia. This complication occurs in $2-10 \%$ of patients receiving chemotherapy regimens with conventional doses. Occasionally, myelosuppression is the main cause of therapy recycling delay with consequent tumor regrowth, severe and prolonged neutropenia, anaemia and thrombocytopenia (2). Neutropenia is associated with an increased number of febrile episodes, increased hospitalization and high costs.

Anaemia and thrombocytopenia involve an increased transfusional need and, subsequently, increased transfusionrelated infective risk and sanitary costs. Thrombocytopenia involves a high haemorrhagic risk (2).

Before chemotherapy, it would be useful to evaluate, the medullary reservoir with the aim of identifying patients subject to severe myelosuppression. One possible way would be to perform an osteomedullary biopsy (OMB) before chemotherapy. However the OMB, being a static image of the medullary function, facilitates the quantitative appraisal, but not the functional appraisal involved in the dynamic recording of what actually occurs in the bone marrow. Moreover, OMB is an invasive test which is often not well-tolerated by the patient.

Less invasive techniques, able to markedly define bone marrow functionality, would be useful in identifying a priori those patients who, after chemotherapy, will have severe and prolonged cytopenias.

A non-invasive method in order to define the medullary reservoir and the risk of prolonged post-chemotherapy aplasia may be represented by the study of reticulocyte fractions and indices and the myeloperoxidase index of neutrophils. 
It is well-known that reticulocytes, in particular, reticulocyte fractions, reflect the state of normal or pathological erythropoiesis (3). Several studies showed that modifications of the reticulocyte indices precede the change of erythroid indices commonly used ( $\mathrm{MCV}, \mathrm{MCH}, \mathrm{CH}$ and $\mathrm{MCHC}$ ) and, are sensitive indices of erythron functional changes (4-9). They may correlate, in dysplastic patients, with a need for erythrocyte and/or platelet transfusion (Giordano G, et al, 39 th Congress of the Italian Society of Hematology, Haematologica (suppl) 88: 26-29, 2003). In patients who underwent bone marrow transplantation, they indicate engraftment and the functional restart of bone marrow, such as a neutrophil increase, but more prematurely (10-15). Reticulocytes may represent the functional order of whole bone marrow and not only of the red cells.

The reticulocyte number, immature reticulocyte fraction, reticulocyte fractions (HFR, MFR and LFR) and reticulocyte indices (MCVr, MCHr, $\mathrm{CHr}$ and $\mathrm{MCHCr}$ ), showing global bone marrow activity, may indicate who will have a severe and long-lasting pancytopenia. The myeloperoxidase index (MPXI) is useful in distinguishing between aplastic and megaloblastic anaemia and in predicting a neutropenic phase after chemotherapy $(16,17)$. From these findings it is inferred that MPXI may correlate not only with the granulocyte function, but also with the global medullar functionality.

Knowing which patients have an increased risk of prolonged cytopenia allows for the dose to be adapted with the aim of minimizing therapy-related myelotoxicity, without loss of antitumoral effectiveness, and consent to maintaining the correct timing of chemotherapy administration. Moreover, it may generate a more appropriate use of growth factors in patients at a higher risk of cytopenia, in order to prevent this. Subsequently, a more targeted use of growth factors may reduce the number of febrile episodes, the use of antibiotics and duration of hospitalization, suggesting an economic benefit (18).

This study aimed to verify whether increased or decreased values of immature reticulocyte fractions (IRF), high, medium and low adsorbance fraction reticulocyte (HFR, MFR and LFR), mean corpuscular volume, mean corpuscular hemoglobin concentration, corpuscular hemoglobin, mean corpuscular hemoglobin of red blood cells and reticulocytes (MCV, $\mathrm{MCH}, \mathrm{CH}, \mathrm{MCHC}$ and $\mathrm{MCVr}, \mathrm{MCHr}, \mathrm{CHr}, \mathrm{MCHCr}$ ), $\mathrm{MCVr} / \mathrm{MCV}, \mathrm{MCHr} / \mathrm{MCH}$ and myeloperoxidase index (MPXI), are related to an increased or decreased risk of therapy-related cytopenia.

\section{Materials and methods}

Patients. The present study is a monocentric, prospective, non-randomized, open-label study. After informed consent according to institutional guidelines, we evaluated 112 patients (or chemotherapy cycles); 82 males and 25 females, responding to inclusion and exclusion criteria, as subsequently reported. Every chemotherapy cycle of each patient was considered as a new case, because our aim was to define a model predicting a priori neutropenia, without regard for number and type of chemotherapy.

The median age was 62 years (range 36-80). Thirty patients were affected by lymphoma and 82 by solid neoplasm. A detailed list of the neoplasia type and chemotherapy regimen is reported in Table I.

Inclusion and exclusion criteria. To verify how disease and therapy interact with bone marrow and influence its kinetics, we enrolled patients, in the study, affected by solid and hematologic neoplasias that are systemic from the onset because of bone marrow direct involvement or by micrometastases (as observed in esophageal, gastric and intestinal neoplasia, small cell lung neoplasia, non-small cell lung neoplasia, breast cancer, prostatic neoplasia, head and neck cancer, neuroblastoma, pancreatic and ampullary cancer, and melanoma) (19-30).

The lack of vitamin B12 and/or folate and/or iron were not regarded as absolute exclusion criteria. An adequate and continual support with B12 and/or folate and/or iron consent a normal bone marrow function. In healthy subjects vitamin B12 presents normal values ranging from 200 to $900 \mathrm{pg} / \mathrm{ml}$. Values $<100 \mathrm{pg} / \mathrm{ml}$ are indicative of a lack of vitamin B12 and are to be supported. In healthy subjects folates present normal values ranging from 6 to $20 \mathrm{ng} / \mathrm{ml}$. Values $<4 \mathrm{ng} / \mathrm{ml}$ are indicative of a lack of folates and are to be supported.

There is the theoretical risk, not supported by literature, that vitamin B12 and folates may sustain the tumoral growth. Thus, it was chosen to administer folates, if necessary, only in solid neoplasia and in lymphomas with a replicative fraction $<50 \%$.

Enrolling patients with inadequate erythropoiesis due to pronounced myelophtisis or myelofibrosis (neutrophil count $<1000 / \mathrm{mm}^{3}$ and/or platelet count $<50000 / \mathrm{mm}^{3}$ and/or hemoglobin concentration $<8 \mathrm{~g} / \mathrm{dl}$ ) was avoided. Cytopenic patients who had radiotherapy to the spine and/or pelvis at least 2 weeks prior to the study were excluded.

Patients with active or recent bleeding, active infections, hypothyroidism, chronic renal failure, hemolytic anaemia, microangiopatic disease, as well as those who received transfusions in the 2 weeks prior to the study, erythropoietin from $<1$ week and myelotoxic drugs at least 1 month before chemotherapy started, were excluded from the study because they may present a falsely high or low reticulocyte count and altered reticulocyte fraction and indices (31-33). Patients affected by gastric carcinoma and, at risk of bleeding, were enrolled in this study only if they were receiving adjuvant chemotherapy. The inclusion and exclusion criteria are listed in Tables II and III.

Automated flow cytochemistry analysis. The tests carried out on the patients are listed in Table IV. Tests were performed as follows: i) 1 or 2 days before chemotherapy administration; ii) 1 day after chemotherapy administration; iii) 3 days after chemotherapy administration (suggested) and iv) 8, 12, 21 and 28 days after chemotherapy administration (suggested, when applicable). Samples were collected as follows: $4 \mathrm{~cm}^{3}$ of whole blood was collected in a test tube with $\mathrm{K}_{2}$ EDTA $1.5-2.2 \mathrm{mg} / \mathrm{ml}$, according to the ICSH proposed reference method for reticulocyte counting (1988). A complete blood count, reticulocyte absolute and percentual count, reticulocyte fractions, immature reticulocyte fraction and reticulocyte indices were obtained by a hematologic automated analyzer Advia $120^{\circledR}$ (Bayer, Diagnostic Division, Tarrytown, NY). 
Table I. Characteristics of the patients concerning diagnosis and chemotherapy regimens administered.

\begin{tabular}{rll}
\hline No. of cases & \multicolumn{1}{c}{ Neoplasm type } & \multicolumn{1}{c}{ Chemotherapy regimen } \\
\hline 26 & Diffuse large B-cell lymphoma & CHOP \\
4 & Hodgkin lymphoma & ABVD \\
2 & Small cell lung cancer & Cisplatinum and etoposide \\
40 & Colon cancer & FOLFOX \\
10 & Hepatocellular carcinoma & GEMOX \\
8 & Non-small cell lung cancer & Cisplatinum and gemcytabine \\
12 & Pancreatic cancer & Gemcytabine and fluorouracil \\
10 & Gastric cancer & Chemotherapy regimens containing fluorouracil \\
\hline
\end{tabular}

Table II. Summary of inclusion criteria.

\begin{tabular}{ll}
\hline 1. Hematological neoplasia & Non-Hodgkin lymphoma \\
& Hodgkin lymphoma \\
& Chronic lymphocytic leukaemia \\
& Multiple myeloma \\
& Gastric cancer \\
& Esophageal cancer \\
& Intestinal cancer \\
& Small cell lung cancer \\
& Non-small cell lung cancer \\
& Breast cancer \\
& Prostatic cancer \\
& Head and neck cancer \\
& Neuroblastoma \\
& Pancreatic and ampullary cancer \\
& Melanoma \\
& Hepatocellular carcinoma \\
& First cycle \\
& Following cycles \\
& $>100$ pg/ml \\
& $<100$ pg/ml, adequately supported \\
& $>4$ ng/ml \\
& $<4$ ng/ml, adequately supported \\
\hline 5. Folates & \\
4. Vitamin B12 &
\end{tabular}

The reticulocyte parameters analysed are: reticulocyte absolute count, reticulocyte percentual count, immature reticulocyte fraction, HFR, MFR, LFR, MCVr, CHr, CHCMr and MPXI.

Statistical analysis. The Chi-square test, Fisher's test and Pearson's correlation coefficient ( $r$ ) and the determination coefficient $\mathrm{R}^{2}$ were used to define a correlation between the collected data and the entity and duration of chemotherapyrelated cytopenia. The risk of cytopenia following chemotherapy was expressed by odds ratio and relative risk.
Table III. Summary of exclusion criteria.

\begin{tabular}{ll}
\hline 1. Neoplasia & $\begin{array}{l}\text { Infiltrating bone marrow } \geq 50 \% \\
\text { Replicative fraction } \geq 50 \%\end{array}$ \\
2. Neutrophil & $<1000 / \mathrm{mm}^{3}$ and \\
3. Platelet & $<50000 / \mathrm{mm}^{3}$ \\
4. Hemoglobin & $<8 \mathrm{~g} / \mathrm{dl}$ \\
5. Radiotherapy & Spine and/or pelvis at least \\
& 2 weeks prior to the study, if \\
& cytopenia is present (as defined \\
& in points 2,3 and 4$)$ \\
Active & Major bleeding in the 2 weeks \\
prior to the study & Active \\
6. Bleeding & Hypothyroidism \\
Hemolytic anemia \\
9icroangiopatic disease \\
13. Infections
\end{tabular}

\section{Results}

Of the 112 cases studied, 60 (54\%), after chemotherapy, had a neutropenic grade of 3 or 4 with an absolute neutrophil count $<500 / \mathrm{mcl}$, for a median of 7 days (range 3-21). Among these 60 cases, $20(33 \%)$ experienced febrile neutropenia.

The statistical analysis of the hematological parameters studied showed that the association of MPXI-positive value and MFR $>10.7 \%$ identified a group of patients with a signi- 
Table IV. Automated flow cytochemistry analysis.

Tests at enrollment

Tests at following cycles
Complete blood count with differential white blood cell count

Reticulocyte counting with reticulocyte fractions and indices

$\mathrm{Na}, \mathrm{K}, \mathrm{Ca}, \mathrm{Mg}$, LDH, GOT, GPT, GGT, total and fractioned bilirubin, alkaline phosphatase, BUN, creatinine, vitamin B12 and folate dosage

Blood iron, ferritin, TIBC

Complete blood count with differential white blood cell count

$\mathrm{Na}, \mathrm{K}, \mathrm{Ca}, \mathrm{Mg}$, LDH, GOT, GPT, GGT, total and fractioned bilirubin, alkaline phosphatase, BUN, creatinine, vitamin B12 and folate dosage

Blood iron, ferritin, TIBC

Erythropoietin dosage

Analysis of urine

Reticulocyte counting with reticulocyte fractions and indices

Homocysteine ficantly lower number of neutropenic events. We assigned a score of 1 to patients with MPXI-positive values and MFR $>10.7 \%(36 / 112,32 \%$ of total $)$ and a score of 0 to the remaining patients $(76 / 112,68 \%$ of total). While only 9 of the 36 patients $(25 \%)$ with a score of 1 developed neutropenia, notably 51 of the 76 patients $(67 \%)$ with a score of 0 showed neutropenic events. These data are statistically significant, with a Chi-square test of 15.760 with $\mathrm{p}<0.0001$, a sensitivity of 0.85 (CI 95\%:0.77-0.91) and a specificity of 0.52 (CI 95\%:0.42-0.59).

The positive predictive value was 0.67 (CI 95\%:0.61-0.72) and the negative predictive value was 0.75 (CI 95\%:0.62-0.85). The odds ratio was 6.3 (CI 95\%:2.5-14.7), with a relative risk of 2.7 (CI 95\%: 1.6-4.8). Taken together, our results suggest that the identification of MPXI and MFR parameters is a rapid, non-invasive, easily standardised, economic method of defining dynamic bone marrow functionality and the risk of prolonged post-chemotherapy neutropenia.

\section{Discussion}

Neutropenia frequently results from the myeloablative effects of systemic chemotherapy. Neutropenia increases the risk of developing serious microbial infections. Bacterial and fungal infections are the major contributing factors to morbidity and mortality in neutropenic patients. Thus, neutropenia frequently causes chemotherapy delay or dose reduction. It may lengthen hospital stay, increase diagnostic and therapeutic costs, and reduce patient quality of life (2).

Colony-stimulating factors (CSFs), such as the granulocyte colony-stimulating factor (G-CSF) and granulocytemacrophage colony-stimulating factor (GM-CSF), are used in the prevention of chemotherapy-related neutropenia. These agents accelerate the formation of neutrophils from committed progenitors, thereby reducing the duration and severity of neutropenia. They reduce the incidence and duration of febrile neutropenia and related hospitalisation. They also consent the reduction of antibiotic use and avoid recycling delay. However, clinical trials have not shown a significant effect of CSFs on overall or disease-free survival, and the high cost of these agents limits their widespread use $(1,2)$.
Further investigation is necessary to determine the positive benefits associated with the use of CSFs. It would be necessary to treat only those patients at a high risk of developing neutropenia with CSFs, in order to show the positive effects of CSFs on the survival of oncologic patients submitted to chemotherapy. Therefore, it is important to develop clinical models which can predict patients at neutropenic risk. These models can help the clinician in detecting patients who qualify to receive CSFs appropriately.

Our study aimed to detect hematological parameters able to identify a priori those patients who, after chemotherapy, will have a severe and prolonged neutropenia. Our intention was to use a non-invasive, effective, rapid and non-expensive method to identify these parameters.

In 1996, Blay and colleagues demonstrated that chemotherapy day-5 lymphocyte count $<700 / \mathrm{mcl}$ was a risk factor for febrile neutropenia (FN) (37). This simple parameter, although useful, is a posteriori data that cannot predict the risk of chemo-related neutropenia before chemotherapy. It does not suggest to the clinician what preventive reduction dose of the chemotherapeutic regimen should be administered in order to avoid neutropenia, its related complications and recycling delay. Bononi and colleagues showed that myeloperoxidase index (MPXI) and large unstained cells (LUC), obtained by the hematologic automated analyser Advia 120 and day-5 lymphopenia were related to post-chemotherapy neutropenia. However, these data predict neutropenia only after chemotherapy administration. In the same study the count of peripheral blood CD $34^{+}$was related to an increased risk of chemotherapy-induced neutropenia. However, the CD $34^{+}$count is expensive and time-consuming (18).

We studied parameters, such as reticulocyte fraction, reticulocyte indices and MPXI, that can be obtained by the hematologic automated analyser Advia 120 at a low cost. Our investigation demonstrated that patients with MPXI-positive value and medium absorbance fraction of reticulocyte (MFR) $>10.7 \%$ showed a significantly lower number of neutropenic events than other patients $(\mathrm{p}<0.0001)$.

It is well-known that reticulocytes, in particular reticulocyte fractions reflect the state of normal or pathological erythropoiesis. Several studies showed that the modifications 
of reticulocyte indices precede the change of erythroid indices commonly used (MCV, $\mathrm{MCH}$ and $\mathrm{MCHC}$ ) and are precocious and sensitive indices of erythron functional changes (10-15).

Reticulocytes correlated in dysplastic patients, with a need for erythrocyte and/or platelet transfusion (4). In patients who had bone marrow transplantation they indicate engraftment and the functional restart of bone marrow, such as a neutrophil increase, earlier. Reticulocyte fractions may represent the function of whole bone marrow and not only of the red cells (7-9). MPXI may correlate not only with the granulocyte function, but also with the global medullary functionality. A study showed that MPXI measurement is a useful tool in the differentiation of megaloblastic from aplastic anaemia (17).

Our intention was to use parameters correlated not only with myeloid lineage, which is the most involved after chemotherapy, but with global bone marrow function. In our study, we enrolled patients not only affected by hematological neoplasms, but also by solid neoplasms that frequently show micrometastases present in bone marrow. This selection was performed in order to obtain, in our cohort of patients, the same interaction between neoplasia and the bone marrow microenvironment without considering the origin of the neoplasia. Our attention was centred on the system constituted by the bone marrow microenvironment, neoplasia with bone marrow involvement and chemotherapy. Since this study aimed to evaluate bone marrow functionality, enrolling patients with pronounced myelophtisis or myelofibrosis was avoided. The major characteristic of this study is the ability to define parameters able to predict neutropenia regardless of the kind of therapy, time of its administration, type of tumor or characteristics of the patient (age, previous chemotherapy or radiotherapy, nutritional status, and individual factors affecting drug metabolism and excretion).

Our analysis of peripheral blood cell parameters has shown that neutropenia correlate with MPXI and MFR. Knowing which patients have an increased risk of prolonged cytopenia before chemotherapy starts, allows for the chemotherapy dose to be adapted to each patient with the aim of minimizing therapy-related myelotoxicity, without loss of antitumoral effectiveness, and consent to maintaining the correct timing of chemotherapy recycling.

It may also generate a more appropriate use of growth factors in patients at a higher risk of developing cytopenia, in order to prevent this. The use of MPXI related to granulopoiesis and MFR related to erythropoiesis, in neutropenia risk definition, may suggest the synergistic use of G-CSF and erythropoietin to prevent chemotherapy-related cytopenia. Previous studies demonstrated no benefits from the association of these growth factors, probably because of a lack of a target population. The intent of a further study, now ongoing in our institute, is to demonstrate the efficacy of growth factors in patients at a high risk of developing neutropenia.

In conclusion, our study shows that MPXI and MFR, obtained by Advia 120 are two economic parameters, easy to obtain in routine complete blood count and useful in identifying patients with a high risk of neutropenia during chemotherapeutic treatment.

\section{References}

1. Dale DC: Colony-stimulating factors for the management of neutropenia in cancer patients. Drugs 62: 1-15, 2002.

2. American Society of Clinical Oncology: Recommendations for the use of hematopoietic colony-stimulating factors: evidencebased, clinical practice guidelines. J Clin Oncol 12: 2471-2508, 1994.

3. Davis BH, Ornvold K and Bigelow NC: Flow cytometric reticulocyte maturity index: a useful laboratory parameter of erythropoietic activity in anemia. Cytometry 15: 35-39, 1995.

4. Breymann C, Bauer C, Major A, Zimmermann R, Gautschi K, Huch A and Huch R: Optimal timing of repeated of rh-erythropoietin administration improves its effectiveness in stimulating erythropoiesis in healthy volunteers. Br J Haematol 93: 295-301, 1996.

5. MacDougall IC, Cavill I, Hulme B, Bain B, McGregor E, McKay P, Saners E, Coles GA and Williams DJ: Detection of functional iron deficiency during erythropoietin treatment: a new approach. Br Med J 304: 225-226, 1992.

6. Brugnara C, Colella GM, Cremins JC, Langley RC, Schneider TJ, Rutherford CJ and Goldberg MA: Effects of subcutaneous recombinant human erythropoietin in normal subjects: development of decreased reticulocyte hemoglobin content and iron deficient erythropoiesis. J Lab Clin Med 123: 660-667, 1994.

7. Chirillo $R$ and Caenaro GF: The evaluation of erythrocyte regeneration by the $\mathrm{H}^{*} 3$ hematology analyzer. In: New Perspective for Hematology - conference proceedings, Bayer Diagnostics, pp40-41, 1993.

8. Brugnara C, Laufer MR, Friedman AJ, Bridges $\mathrm{K}$ and Platt O: Reticulocyte hemoglobin content $(\mathrm{CHr})$ : early indicator of iron deficiency and response to therapy. Blood 83: 3100-3101, 1994.

9. Mohandas N: Reticulocyte biology in red blood cell disorders. In: New Perspective for Hematology - conference proceedings, Bayer Diagnostics, pp46-47, 1993.

10. Greinix HT, Linkesch W, Keil F, Kalhs P, Schwarzinger I, Schneider B, Oesterreicher C, Brugger S, Kapiotis S and Lechner K: Early detection of hematopoietic engraftment after bone marrow and peripheral blood stem cell transplantation by highly fluorescent reticulocyte counts. Bone Marrow Transplant 14: 307-313, 1994.

11. Lesesve JF, Lacombe F, Boisseau MR and Bernard P: Comparative study of the differential white blood cell count using three automated analyzers: Coulter STKS, Sysmex NE 8000 and Technicon H-1. Ann Biol Clin 524: 295-297, 1994.

12. Kessinger A, Armitage JO, Landmark JD, Smith DM and Weisenburger DD: Autologous peripheral hematopoietic stem cell transplantation restores hematopoietic function following marrow ablative therapy. Blood 71: 723-727, 1988.

13. Arnold R, Schmeiser T, Heit W, Frickhofen N, Pabst G, Heimpel $\mathrm{H}$ and Kubanek B: Hemopoietic reconstitution after bone marrow transplantation. Exp Hematol 14: 271-277, 1986.

14. d'Onofrio G, Tichelli A, Foures C and Theodorsen L: Indicators of haematopoietic recovery after bone marrow transplantation: the role of reticulocyte measurements. Clin Lab Haematol 18: S45-S53, 1996.

15. Dalal BI, Stockford GR, Naiman SC, Spinelli JJ and Phillips GL: Criteria for marrow engraftment: comparison of reticulocyte maturity index with conventional parameters. Bone Marrow Transplant 17: 91-92, 1996.

16. Ziaei JE and Dastgiri S: Role of myeloperoxidase index in differentiation of megaloblastic and aplastic anemia. Indian J Med Sci 58: 345-348, 2004.

17. Bononi A, Lanza F, Dabusti M, Gusella M, Gilli G, Menon D, Toso S, Crepaldi G, Marenda B, Abbasciano V and Ferrazzi E: Increased myeloperoxidase index and large unstained cell values can predict the neutropenia phase of cancer patients treated with standard dose chemotherapy. Cytometry 46: 92$97,2001$.

18. Dempke W, Von Poblozki A, Grothey A and Schmoll HJ: Human hematopoietic growth factors: old lessons and new perspectives. Anticancer Res 20: 5155-5164, 2000.

19. Inoue H, Kajiyama Y and Tsurumaru M: Clinical significance of bone marrow micrometastases in esophageal cancer. Dis Esophagus 17: 328-332, 2004.

20. Skinner LJ, Conlon BJ, Russell JD, O'Sullivan GC and O'Dwyer TP: Detection of bone marrow micrometastases in the rib marrow of head and neck cancer patients: a prospective pilot study. Eur Arch Otorhinolaryngol 262: 103-106, 2005. 
21. Yu JJ, Brennan M, Christos P, Osborne MP, Hoda S and Simmons RM: Bone marrow micrometastases and adjuvant treatment of breast cancer. Breast J 10: 181-185, 2004.

22. Ryan P, McCarthy S, Kelly J, Collins JK, Dunne C, Grogan L, Breathnach O, Shanahan F, Carey PD, Walsh TN and O'Sullivan GC: Prevalence of bone marrow micrometastases in esophagogastric cancer patients with and without neoadjuvant chemoradiotherapy. J Surg Res 117: 121-126, 2004.

23. Tsang KS, Li CK, Tsoi WC, Leung Y, Shing MM, Chik KW, Lee V, Ng MH and Yuen PM: Detection of micrometastasis of neuroblastoma to bone marrow and tumor dissemination to hematopoietic autografts using flow cytometry and reverse transcriptase-polymerase chain reaction. Cancer 97: 2887-2897, 2003.

24. Capov I: Diagnosis of bone micrometastases in non-small cell lung carcinoma - initial results. Rozhl Chir 82: 145-148, 2003.

25. Bjornland K, Flatmark K, Mala T, Mathisen O, Bakka A, Aasen AO, Bergan A, Soreide O and Fodstad O: Detection of disseminated tumour cells in bone marrow of patients with isolated liver metastases from colorectal cancer. J Surg Oncol 82: 224-227, 2003.

26. Passlick B: Micrometastases in non-small cell lung cancer (NSCLC). Lung Cancer 34: S25-S29, 2001.

27. Van Heek NT, Tascilar M, Van Beekveld JL, Drillenburg P, Offerhaus GJ and Gouma DJ: Micrometastases in bone marrow of patients with suspected pancreatic and ampullary cancer. Eur J Surg Oncol 27: 740-745, 2001.

28. Vagunda V, Landys K, Kankkunnen JP, Vagundova M, Hultborn R, Kovarik J and Hugosson J: Bone marrow micrometastases in patients with stage I-II localised prostate cancer. Eur J Cancer 37: 1847-1852, 2001.

29. Ghossein RA, Carusone L and Bhattacharya S: Molecular detection of micrometastases and circulating tumor cells in melanoma, prostatic and breast carcinomas. In Vivo 14: 237-250, 2000 .
30. Pelasi G, Pasini F, Pavanel F, Bresaola E, Schiavon I and Iannucci A: Effects of different immunolabeling techniques on the detection of small-cell lung cancer cells in bone marrow. J Histochem Cytochem 47: 1075-1088, 1999.

31. Fourcade C, Jary L and Belaouni H: Reticulocyte analysis provided by the coulter GEN.S: significance and interpretation in regenerative and non-regenerative hematologic conditions. Lab Hematol 5: 153-158, 1999.

32. Lin CK, Hsu HC and Chay WK: Reticulocyte counts with maturation fractions in pancytopenic evaluation by a fully automated cell counter. J Clin Lab Anal 7: 371-375, 1993.

33. Chang $\mathrm{C}$ and Kass L: Clinical significance of immature reticulocyte fraction determined by automated reticulocyte counting. Am J Clin Pathol 108: 69-73, 1997.

34. Coiffier B, Gisselbrecht C, Herbrecht R, et al: LNH-84 regimen: a multicenter study of intensive chemotherapy in 737 patients with aggressive malignant lymphoma. J Clin Oncol 7: 1018-1026, 1989.

35. Velasquez W, Cabanillas F, Salvador P, et al: Effective salvage therapy for lymphoma with cisplatin in combination with high dose Ara C and dexamethasone (DHAP). Blood 72: 117-122, 1988.

36. Nichols C, Williams S, Loehrer PJ, et al: A randomized study of cisplatin dose intensity in poor risk germ cell tumors: a Southeastern Cancer Study Group and Southwest Oncology Group protocol. J Clin Oncol 9: 1163-1172, 1991.

37. Blay JY, Chauvin F, Le Cesne A, et al: Early lymphopenia after cytotoxic chemotherapy as a risk factor for febrile neutropenia. J Clin Oncol 6: 1377-1387, 1996. 